\title{
POINCARÉ SHEAVES ON THE MODULI SPACES OF TORSIONFREE SHEAVES OVER AN IRREDUCIBLE CURVE
}

\author{
USHA N. BHOSLE AND INDRANIL BISWAS
}

\begin{abstract}
Let $Y$ be a geometrically irreducible reduced projective curve defined over $\mathbb{R}$. Let $U_{Y}(n, d)$ (respectively, $U_{Y}^{\prime}(n, d)$ ) be the moduli space of geometrically stable torsionfree sheaves (respectively, locally free sheaves) on $Y$ of rank $n$ and degree $d$. Define $\chi=d+n(1-\operatorname{genus}(Y))$, where genus $(Y)$ is the arithmetic genus. If $2 n$ is coprime to $\chi$, then there is a Poincaré sheaf over $U_{Y}(n, d) \times Y$. If $2 n$ is not coprime to $\chi$, then there is no Poincaré sheaf over any nonempty open subset of $U_{Y}^{\prime}(n, d)$.
\end{abstract}

\section{INTRODUCTION}

Let $C$ be a smooth complex projective curve. Let $U_{C}(n, d)$ be the moduli space of stable vector bundles over $C$ of rank $n$ and degree $d$. Assume that $U_{C}(n, d)$ is nonempty; this is ensured if genus $(C) \geq 2$. A vector bundle $\mathcal{E} \longrightarrow U_{C}(n, d) \times C$ is called a Poincaré vector bundle if for each point $z \in U_{C}(n, d)$, the vector bundle $\left.\mathcal{E}\right|_{\{z\} \times C}$ over $C$ is in the isomorphism class defined by $z$. By a Poincaré vector bundle over an open subset $\mathcal{U} \subset U_{C}(n, d)$ we will mean a vector bundle $\mathcal{E} \longrightarrow \mathcal{U} \times C$ such that for each point $z \in \mathcal{U}$, the vector bundle $\left.\mathcal{E}\right|_{\{z\} \times C}$ is in the isomorphism class defined by $z$.

If $n$ is coprime to $d$, then there is a Poincare vector bundle over $U_{C}(n, d) \times C$. If $n$ and $d$ are not coprime, then there is no Poincaré vector bundle over any nonempty open subset of $U_{C}(n, d)$ [Ra, Theorem 2]. We note that $n$ and $d$ are coprime if and only if $n$ and $\chi:=d-n(\operatorname{genus}(C)-1)$ are coprime; the Euler characteristic of any vector bundle of rank $n$ and degree $d$ over $C$ is $\chi$.

Let $D$ be a geometrically irreducible smooth projective curve defined over $\mathbb{R}$. A vector bundle $E$ over $D$ is called geometrically stable if the vector bundle $E \bigotimes_{\mathbb{R}} \mathbb{C}$ over $D \times_{\mathbb{R}} \mathbb{C}$ is stable. Let $U_{D}(n, d)$ be the moduli space of geometrically stable vector bundles over $C$ of rank $n$ and degree $d$. Assume that $U_{D}(n, d)$ is nonempty; this is ensured if genus $(D) \geq 2$. If $D$ has a real point, then there is a Poincaré vector bundle over $U_{D}(n, d) \times D$ if and only if $n$ and $\chi$ are coprime, where $\chi$ is defined as above. If $D$ does not have any real point, then there is a Poincaré vector bundle over $U_{D}(n, d) \times D$ if and only if $2 n$ and $\chi$ are coprime. (See $[\mathrm{BiHu}]$.)

Our aim here is to address this question for curves not necessarily smooth.

Let $Y$ be a geometrically irreducible reduced projective curve defined over the real numbers. A torsionfree sheaf $V$ on $Y$ is called geometrically stable if the coherent sheaf

2000 Mathematics Subject Classification. 14F05, 14D20, 14P99.

Key words and phrases. Poincaré sheaf, descent condition, stable sheaf, real curve. 
on $Y \times_{\mathbb{R}} \mathbb{C}$ defined by $V$ is stable. Let $U_{Y}(n, d)$ (respectively, $U_{Y}^{\prime}(n, d)$ ) be the moduli space of geometrically stable torsionfree sheaves (respectively, locally free sheaves) on $Y$ of rank $n$ and degree $d$. We assume that the moduli space $U_{Y}(n, d)$ has points defined over $\mathbb{C}$ (the set of points of $U_{Y}(n, d)$ defined over $\mathbb{R}$ is allowed to be empty); this is ensured if the arithmetic genus of $Y$ is at least two.

Define $\chi:=d-n(\operatorname{genus}(Y)-1)$, which is the Euler characteristic of any sheaf on $Y$ lying in $U_{Y}(n, d)$.

Assume that $Y$ does not have any point defined over $\mathbb{R}$.. We prove the following (see Theorem 3.2):

If $2 n$ is coprime to $\chi$, then there is a Poincaré sheaf over $U_{Y}(n, d) \times Y$.

If $2 n$ is not coprime to $\chi$, then there is no Poincaré sheaf over any nonempty open subset of $U_{Y}^{\prime}(n, d)$.

The proof of Theorem 3.2 given here is different from the one in $\mathrm{BiHu}$ ] where it is proved under the assumption that $Y$ is smooth. The proof in [BiHu] uses the smoothness assumption in an essential way.

\section{Poincaré SheAF}

Let $X$ be an irreducible reduced projective curve defined over an algebraically closed field $k$ of characteristic zero. Let $U(n, d)$ be the moduli space of torsionfree stable sheaves of rank $n$ and degree $d$ on $X$; it is a GIT quotient of a Quot scheme $Q$ of coherent quotient sheaves of $\mathcal{O}_{X}^{N}$ by $\operatorname{PGL}(N, k)[\mathrm{New}$, [Se]. We assume that $U(n, d)$ has points defined over $\mathbb{C}$; as mentioned before, the set of points of $U_{Y}(n, d)$ defined over $\mathbb{R}$ may be be empty.

Let $Q^{\prime}$ denote the set of points of $Q$ corresponding to stable quotient sheaves. Over $Q^{\prime} \times X$, there is a universal sheaf

$$
\mathcal{E} \longrightarrow Q^{\prime} \times X
$$

Let $\mathcal{E}_{q}:=\left.\mathcal{E}\right|_{q \times X}$.

The points of $Q^{\prime} \subset Q$ are identified with the properly stable points for the action of $\operatorname{PGL}(N, k)$ on $Q$. The isotropy subgroup $G_{q}$ at $q$ is isomorphic to $\operatorname{Aut}\left(\mathcal{E}_{q}\right)$. Since $\mathcal{E}_{q}$ is stable, we have $G_{q}=k^{*}$; any $c \in k^{*}$ acts on $\mathcal{E}$ by multiplication with the scalar $c$. By a result of Nevins [Nev, p. 2482, Theorem 1.2], the sheaf $\mathcal{E}$ descends to $U(n, d) \times X$ if and only if for every $(q, x) \in Q^{\prime} \times X$, the $\mathcal{O}_{Q^{\prime} \times X,(q, x)}$-modules

$$
\mathcal{E} \otimes\left(\mathcal{O}_{Q^{\prime} \times X} / m_{q, x}\right) \quad \text { and } \operatorname{Tor}_{1}^{\mathcal{O}_{Q^{\prime} \times X}}\left(\mathcal{E}, \mathcal{O}_{Q^{\prime} \times X} / m_{q, x}\right)
$$

are trivial representations of $G_{q}$ (the action of $G_{q}$ on $X$ is taken to be the trivial one); here $m_{q, x}$ denotes the ideal sheaf of the point $(q, x) \in Q^{\prime} \times X$.

The proof of Proposition 2.1 is straightforward.

Proposition 2.1. Any $c \in k^{*}$ acts on the modules

$$
\mathcal{E} \otimes\left(\mathcal{O}_{Q^{\prime} \times X / m_{q, x}}\right) \quad \text { and } \quad \operatorname{Tor}_{1}^{\mathcal{O}_{Q^{\prime} \times X}}\left(\mathcal{E}, \mathcal{O}_{Q^{\prime} \times X} / m_{q, x}\right)
$$


by multiplication by the scalar $c$.

Proposition 2.2. (1) There exists a Poincaré sheaf on $U(n, d) \times X$ if $n$ and $d$ are coprime.

(2) There is no Poincaré sheaf on any open subset of $U^{\prime}(n, d)$ if $n$ and $d$ are not coprime.

Proof. The first part is proved in $\left[\mathrm{New}\right.$, Ch. $5, \S 7$, Theorem $\left.5.12^{\prime}\right]$. We include a proof which will be referred in Section 3 ,

Let $\chi:=\chi\left(E_{q}\right)$, where $q \in Q^{\prime}$. Since g.c.d. $(n, d)=1$, there exist integers $a$ and $b$ such that $n a+\chi b=-1$. Let $\operatorname{Det}(\mathcal{E})$ be the determinant line bundle on $Q^{\prime}$ associated to the family $\mathcal{E} \longrightarrow Q^{\prime} \times X$. We recall that

$$
\operatorname{Det}(\mathcal{E})=\left(\operatorname{det} R^{0} f_{*} \mathcal{E}\right) \otimes\left(\operatorname{det} R^{1} f_{*} \mathcal{E}\right)^{*},
$$

where $f: Q^{\prime} \times X \longrightarrow Q^{\prime}$ is the projection. Fix a smooth point $x_{0} \in X$. Define the line bundle on $Q^{\prime}$

$$
\mathcal{L}:=(\operatorname{Det}(\mathcal{E}))^{b} \otimes\left(\left(\left.\Lambda^{n} \mathcal{E}\right|_{Q^{\prime} \times x_{0}}\right)^{\otimes a}\right) .
$$

Any $c \in k^{*}$ acts on $\mathcal{L}$ as multiplication by $c^{-1}$. Define

$$
\mathcal{E}^{\prime}:=\mathcal{E} \otimes p_{Q^{\prime}}^{*} \mathcal{L}
$$

Then, by Proposition 2.1, the group $k^{*}$ acts trivially on

$$
\mathcal{E}^{\prime} \otimes\left(\mathcal{O}_{Q^{\prime} \times X} / m_{q, x}\right) \quad \text { and } \operatorname{Tor}_{1}^{\mathcal{O}_{Q^{\prime} \times X}}\left(\mathcal{E}^{\prime}, \mathcal{O}_{Q^{\prime} \times X} / m_{q, x}\right) .
$$

By a result of Nevins [Nev, Theorem 1.2], the sheaf $\mathcal{E}^{\prime}$ descends to $U(n, d) \times X$ giving the required Poincaré sheaf.

Th second part follows exactly as in [BiHo, Corollary 2.3].

\section{Curves defined over Real numbers}

Let $Y$ be a geometrically irreducible reduced curve defined over $\mathbb{R}$. Let $U_{Y}(n, d)$ be the moduli space of geometrically stable torsionfree sheaves on $Y$ of rank $n$ and degree $d$. We assume that $U_{Y}(n, d)$ is nonempty. Let

$$
U_{Y}^{\prime}(n, d) \subset U_{Y}(n, d)
$$

be the Zariski open subscheme parametrizing the locally free stable sheaves.

Lemma 3.1. Assume that $Y$ has a smooth real point. Then there is a Poincaré sheaf on $U_{Y}(n, d) \times Y$ if $d$ is coprime to $n$. If $d$ is not coprime to $n$, then there is no Poincaré sheaf on any nonempty Zariski open subset of $U_{Y}^{\prime}(n, d)$.

Proof. Let $X:=Y \times_{\mathbb{R}} \mathbb{C}$ be the complex curve obtained by base change to $\mathbb{C}$. We note that the base change $U_{Y}(n, d)_{\mathbb{C}}=U_{Y}(n, d) \times_{\mathbb{R}} \mathbb{C}$ is the moduli space $U_{X}(n, d)$ of stable torsionfree sheaves on $X$ of rank $n$ and degree $d$. Similarly, $U_{Y}^{\prime}(n, d)_{\mathbb{C}}=U_{Y}^{\prime}(n, d) \times_{\mathbb{R}} \mathbb{C}$ is the moduli space $U_{X}^{\prime}(n, d)$ of stable vector bundles on $X$ of rank $n$ and degree $d$. 
If $\mathcal{E} \longrightarrow \mathcal{U} \times Y$ is a Poincaré sheaf, where $\mathcal{U} \subset U_{Y}^{\prime}(n, d)$ is a nonempty Zariski open subset, then $\mathcal{E} \otimes_{\mathbb{R}} \mathbb{C}$ is a Poincaré sheaf on $\left(\mathcal{U} \times_{\mathbb{R}} \mathbb{C}\right) \times X$. In that case, Proposition $2.2(2)$ says that $d$ is coprime to $n$.

Conversely, if $d$ is coprime to $n$, taking the point $x_{0}$ in the proof of Proposition $2.2(1)$ to be a real point we see that the Poincaré sheaf constructed in the proof of Proposition 2.2 is defined over $\mathbb{R}$.

Henceforth, we assume that $Y$ does not have any real point.

As in Section 2, define

$$
\chi:=d+n(1-\operatorname{genus}(Y)),
$$

where $\operatorname{genus}(Y)$ is the arithmetic genus; note that $\chi=\chi(E)$ for any $E \in U_{Y}(n, d)$.

Theorem 3.2. Assume that $Y$ does not have any point defined over $\mathbb{R}$.

(1) If $2 n$ is coprime to $\chi$, then there is a Poincaré sheaf over $U_{Y}(n, d) \times Y$.

(2) If $2 n$ is not coprime to $\chi$, then there is no Poincaré sheaf over any nonempty open subset of $U_{Y}^{\prime}(n, d)$.

Proof. For a variety $Z$ defined over $\mathbb{C}$, let

$$
\bar{Z}:=\left(Z(\mathbb{C}), \overline{\mathcal{O}}_{Z}\right)
$$

be the complex conjugate variety of $Z$. As in the proof of Lemma 3.1 , define $X:=Y \times_{\mathbb{R}} \mathbb{C}$. Let

$$
\sigma: X \longrightarrow \bar{X}
$$

be the natural isomorphism obtained from the fact that $X$ is the base change of $Y$ to $\mathbb{C}$. The composition

$$
X \stackrel{\sigma}{\longrightarrow} \bar{X} \stackrel{\bar{\sigma}}{\longrightarrow} \overline{\bar{X}}=X
$$

is the identity map of $X$.

First assume that $2 n$ is coprime to $\chi$. Fix a smooth effective real divisor $D$ on $Y$ of degree two. We note that such divisors exist; they are in bijective correspondence with the pairs of smooth points of $X$ of the form $\{x, \sigma(x)\}$, where $\sigma$ is the map in (3.2).

That there is a Poincaré sheaf over $U_{Y}(n, d) \times Y$ can be shown exactly as done in the proof of the first part of Proposition 2.2. Instead of the smooth point $x_{0}$ in the proof of Proposition 2.2, we take the above divisor $D$. More precisely, replace $\left.\Lambda^{n} \mathcal{E}\right|_{Q^{\prime} \times x_{0}}$ in (2.1) by the line bundle $\left.\Lambda^{2 n} \mathcal{E}\right|_{Q^{\prime} \times D}$; note that $\left.\mathcal{E}\right|_{Q^{\prime} \times D}$ is a vector bundle of rank $2 n$ over $Q^{\prime}$. Since $2 n$ is coprime to $\chi$ the rest of the argument remains unchanged.

Now assume that $\chi$ is not coprime to $2 n$.

The Euler characteristic $\chi$ is coprime to $n$ if and only if $d$ is coprime to $n$. Therefore, if $\chi$ is not coprime to $n$, then from Proposition 2.2 we know that there is no Poincaré sheaf on any nonempty Zariski open subset of $U_{Y}^{\prime}(n, d)$; note that any Poincaré sheaf on $\mathcal{U} \times Y$ defines a Poincaré sheaf on $\left(\mathcal{U} \times_{\mathbb{R}} \mathbb{C}\right) \times X$ by base change to $\mathbb{C}$. 
Consequently, we assume $\chi$ is coprime to $n$.

Since $\chi$ is not coprime to $2 n$, we conclude that $\chi$ is even. Therefore, $n$ is odd because $\chi$ is coprime to $n$. Define

$$
b_{0}:=(n-1) / 2 \in \mathbb{Z} .
$$

There is an integer $\delta$ and a real point

$$
L_{0} \in \operatorname{Pic}^{\delta}(Y)
$$

such that $L_{0}$ is not a line bundle over $Y$ [BK, p. 226, Corollary 2] (see [GH, p. 159, Proposition 2.2(2)] for smooth $Y$ ). Since there are line bundles on $Y$ of degree two (recall that there is a smooth real divisor of degree two), for any integer $m$, we have the real point

$$
L_{0} \otimes \xi^{\otimes m} \in \operatorname{Pic}^{\delta+2 m}(Y),
$$

where $\xi \longrightarrow Y$ is a line bundle of degree two, which is not a line bundle over $Y$.

We will consider $L_{0}$ as a line bundle over $X$, because it defines a point of $\operatorname{Pic}^{\delta+2 m}(X)$. Note that $H^{i}\left(X, L_{0}\right)$ is of even dimension because it has a quaternionic structure. Hence $\chi\left(L_{0}\right)$ is even. Therefore, for any even integer $b$, there is a real point of $\operatorname{Pic}^{b-1+\operatorname{genus}(Y)}(Y)$ which is not a line bundle over $Y$; note that the Euler characteristic is $b$.

We may assume that the degree $d$ is sufficiently large positive by tensoring with the line bundle $\mathcal{O}_{Y}(a D)$, where $D$ as before is a real smooth effective divisor of degree two, and $a \in \mathbb{N}$. Note that $\chi$ is also sufficiently large positive because $\chi=d-n$ (genus $(Y)-1)$.

We noted earlier that $n$ is odd, and $\chi$ is even. Hence

$$
\chi+n(\operatorname{genus}(Y)-1)+1-\operatorname{genus}(Y) \equiv 0 \bmod 2 .
$$

We also noted above that for any even integer $b$, there is a real point of $\operatorname{Pic}^{b-1+\operatorname{genus}(Y)}(Y)$ which is not a line bundle over $Y$. Hence from (3.5) we conclude that there is a real point

$$
L \in \operatorname{Pic}^{\chi+n(\operatorname{genus}(Y)-1)}(Y)=\operatorname{Pic}^{d}(Y)
$$

which is not a real line bundle over $Y$. Fix such a point $L$.

The line bundle over $X$ (respectively, $\bar{X}$ ) (see (3.3)) corresponding to $L$ in (3.6) will be denoted by $L$ (respectively, $\bar{L}$ ). Since $L$ in (3.6) is a real point, but not a line bundle on $Y$, there is a unique isomorphism

$$
\eta: L \longrightarrow \sigma^{*} \bar{L}
$$

such that $\left(\sigma^{*} \bar{\eta}\right) \circ \eta=-\operatorname{Id}_{L}$. (see (3.3)).

Let

$$
T: \mathcal{O}_{X} \oplus \mathcal{O}_{X} \longrightarrow \mathcal{O}_{\bar{X}} \oplus \mathcal{O}_{\bar{X}}
$$

be the isomorphism defined by $\left(f_{1}, f_{2}\right) \longmapsto\left(-f_{2} \circ \sigma^{-1}, f_{1} \circ \sigma^{-1}\right)$. We note that

$$
\left(\sigma^{*} \bar{T}\right) \circ T=-\operatorname{Id}_{\mathcal{O}_{X}^{\oplus 2}} .
$$


Consider $b_{0}$ defined in (3.4). Elements of $H^{1}\left(X, L^{\vee} \otimes\left(\mathcal{O}_{X}^{\oplus 2}\right)\right)^{\oplus b_{0}}$ parametrize extensions of the form

$$
0 \longrightarrow\left(\mathcal{O}_{X}^{\oplus 2}\right)^{\oplus b_{0}} \longrightarrow V \longrightarrow L \longrightarrow 0,
$$

where $L$ is the above line bundle on $X$. There is a universal short exact sequence

$$
0 \longrightarrow\left(\mathcal{O}_{X \times \mathbb{A}}^{\oplus 2}\right)^{\oplus b_{0}} \longrightarrow \mathcal{V} \longrightarrow p_{1}^{*} L \longrightarrow 0
$$

where $\mathbb{A}:=H^{1}\left(X, L^{\vee} \otimes\left(\mathcal{O}_{X}^{\oplus 2}\right)\right)^{\oplus b_{0}}$, and $p_{1}$ is the projection of $X \times \mathbb{A}$ to $X$. Since $d$ is sufficiently large, all stable vector bundles over $X$ of rank $n$ and determinant $L$ occur in the family $\mathcal{V}$ in (3.9) . Let

$$
\mathcal{S} \subset \mathbb{A}
$$

be the locus of stable bundles for the family in (3.9); from the openness of the stability condition (see [Ma]) it follows that $\mathcal{S}$ is a Zariski open subset. Let $U_{X}^{\prime}(n, L)$ be the moduli space of stable vector bundles $E$ over $X$ of rank $n$ with $\bigwedge^{n} E=L$. Let

$$
\Phi: \mathcal{S} \longrightarrow U_{X}^{\prime}(n, L)
$$

be the surjective morphism representing the family $\mathcal{V}$ in (3.9).

Let

$$
\psi: U_{X}^{\prime}(n, L) \longrightarrow U_{X}^{\prime}(n, L)
$$

be the bijection defined by $E \longmapsto \sigma^{*} \bar{E}$, where $\sigma$ is the isomorphism in (3.2), and $\bar{E}$ is the vector bundle over $\bar{X}$ corresponding to the vector bundle $E$ over $X$. It should be clarified that $\psi$ is not algebraic, it is not even holomorphic, but anti-holomorphic. Let $\overline{U_{X}^{\prime}(n, L)}$ be the variety obtained from $U_{X}^{\prime}(n, L)$ using the automorphism of the field $\mathbb{C}$ defined by $z \longmapsto \bar{z}$. Therefore, the complex points of $\overline{U_{X}^{\prime}(n, L)}$ are in bijective correspondence with the complex points of $U_{X}^{\prime}(n, L)$. Using this bijection, if we consider $\psi$ in (3.12) as a map $U_{X}^{\prime}(n, L) \longrightarrow \overline{U_{X}^{\prime}(n, L)}$, then this map is an algebraic isomorphism.

The isomorphism $\psi$ in (3.12) is clearly involutive. This $\psi$ defines a real structure on the complex variety $U_{X}^{\prime}(n, L)$. The corresponding variety over $\mathbb{R}$ is the moduli space $U_{Y}^{\prime}(n, L):=\operatorname{det}^{-1}(L)$, where

$$
\text { det }: U^{\prime}(n, d) \longrightarrow \operatorname{Pic}^{d}(Y)
$$

is the morphism defined by $E \longmapsto \bigwedge^{n} E$, and $L$ is the point in (3.6).

Let

$$
\theta: H^{1}\left(X, L^{\vee} \otimes\left(\mathcal{O}_{X}^{\oplus 2}\right)\right)^{\oplus b_{0}} \longrightarrow H^{1}\left(X, L^{\vee} \otimes\left(\mathcal{O}_{X}^{\oplus 2}\right)\right)^{\oplus b_{0}}
$$

be the conjugate linear involution constructed using $\eta$ and $T$ defined in (3.7) and (3.8) respectively. The subset $\mathcal{S}$ in (3.10) is preserved by $\theta$, and

$$
\psi \circ \Phi=\Phi \circ \theta
$$

where $\Phi$ and $\psi$ are constructed in (3.11) and (3.12) respectively. Therefore, the morphism $\Phi$ is defined over $\mathbb{R}$. 
The fixed point locus

$$
\mathbb{A}^{\theta} \subset \mathbb{A}
$$

for $\theta$ is a $\mathbb{R}$-linear subspace such that the natural homomorphism

$$
\mathbb{A}^{\theta} \otimes_{\mathbb{R}} \mathbb{C} \longrightarrow H^{1}\left(X, L^{\vee} \otimes\left(\mathcal{O}_{X}^{\oplus 2}\right)\right)^{\oplus b_{0}}
$$

is an isomorphism; hence $\mathbb{A}^{\theta}$ is Zariski dense in $\mathbb{A}$.

Let

$$
\mathcal{W} \longrightarrow \mathcal{U} \times Y
$$

be a Poincaré sheaf, where $\mathcal{U}$ is a nonempty Zariski open subset of $U_{Y}^{\prime}(n, d)$. The morphism det in (3.13) is an open smooth surjective morphism, hence the image $\operatorname{det}(\mathcal{U})$ is a nonempty Zariski open subset of $\operatorname{Pic}^{d}(Y)$. We noted earlier that there is a real point of $\operatorname{Pic}^{d}(Y)$ which are not a line bundle on $Y$ (see (3.6) ), and also it is known that any (nonempty) connected component of the locus of real points in a smooth quasiprojective variety is Zariski dense. Hence the set of real points of $\operatorname{Pic}^{d}(Y)$ which are not line bundles on $Y$ is Zariski dense in $\operatorname{Pic}^{d}(Y)$. In particular, this set intersects the Zariski open subset $\operatorname{det}(\mathcal{U})$, where $\mathcal{U}$ is the open subset in (3.17). Therefore, we may take the chosen point $L$ in (3.6) to be inside $\operatorname{det}(\mathcal{U})$. Hence we assume that

$$
L \in \operatorname{det}(\mathcal{U})
$$

Since $\mathcal{S}$ in (3.10) is a nonempty Zariski open subset, and $\mathbb{A}^{\theta}$ defined in (3.16) is Zariski dense, we know that $\mathcal{S} \cap \mathbb{A}^{\theta}$ is Zariski dense in $\mathbb{A}$. Take any point

$$
z \in \mathcal{S} \cap \mathbb{A}^{\theta}
$$

such that the corresponding vector bundle

$$
\mathcal{V}_{z}:=\left.\mathcal{V}\right|_{\{z\} \times X}
$$

lies in the open subset $\mathcal{U}$ in (3.17). Since $z \in \mathbb{A}^{\theta}$, from (3.15) we know that $\psi(\Phi(z))=$ $\Phi(z)$, where $\psi$ and $\Phi$ are defined in (3.12) and (3.11) respectively. Therefore, there is an isomorphism

$$
\beta: \mathcal{V}_{z} \longrightarrow \sigma^{*} \overline{\mathcal{V}_{z}}
$$

constructed using $\sigma$ and $T$ defined in (3.2) and (3.8) respectively, such that

$$
\left(\sigma^{*} \bar{\beta}\right) \circ \beta=-\operatorname{Id}_{\mathcal{V}_{z}} .
$$

This isomorphism $\beta$ fits in the following commutative diagram

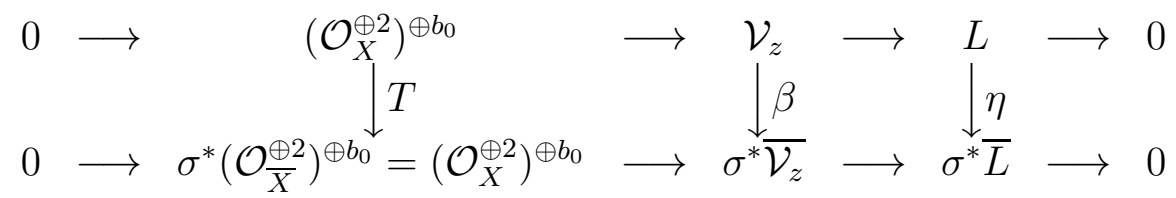

where the horizontal exact sequences are as in (3.9), and the maps $T$ and $\eta$ are constructed in (3.8) and (3.7) respectively. 
Let $\underline{z} \in U^{\prime}(n, d)$ be the point corresponding to the vector bundle $\mathcal{V}_{z}$ in (3.19). Restricting the Poincaré bundle $\mathcal{W}$ to $\{\underline{z}\} \times Y$ we get a vector bundle over $Y$ which is represented by this point $\underline{z}$ of the moduli space.

For any geometrically stable vector bundle $W_{0}$ over $Y$, the group of all automorphisms of the corresponding vector bundle $W$ over $X$ is the group of nonzero complex numbers. There is a natural isomorphism

$$
\gamma: W \longrightarrow \sigma^{*} \bar{W}
$$

such that $\left(\sigma^{*} \bar{\gamma}\right) \circ \gamma=\operatorname{Id}_{W}$. Since any other isomorphism $W \longrightarrow \sigma^{*} \bar{W}$ must be of the form $c \cdot \gamma$, where $c \in \mathbb{C}^{*}$ (recall that the automorphisms of $W$ are the nonzero scalars), we conclude that there is no isomorphism

$$
\phi: W \longrightarrow \sigma^{*} \bar{W}
$$

such that $\left(\sigma^{*} \bar{\phi}\right) \circ \phi=-\operatorname{Id}_{W}$ (there is no complex number such that $\bar{c} c=-1$ ).

But the vector bundle $\left.\mathcal{W}\right|_{\{\underline{z}\} \times Y}$ in (3.17) contradicts the above observation that it is not possible to have simultaneously isomorphisms $\gamma$ and $\phi$ of the above type. From this contradiction we conclude that there is no Poincaré sheaf over $\mathcal{U} \times Y$. This completes the proof of the theorem.

\section{REFERENCES}

[BK] E. Ballico and J. Kollár, The Picard group of singular curves, Abh. Math. Sem. Univ. Hamburg 73 (2003), 225-227.

[BiHo] I. Biswas and N. Hoffmann, Poincaré families and automorphisms of principal bundles on a curve, C.R. Acad. Sci. Paris 347 (2009), 1285-1288.

[BiHu] I. Biswas and J. Hurtubise, Universal vector bundle over the reals, Trans. Amer. Math. Soc. (to appear), http://arxiv.org/abs/0909.0041.

[GH] B. Gross and J. Harris, Real algebraic curves, Ann. Sci. Éc. Norm. Sup. 14 (1981), 157-182.

[Ma] M. Maruyama, Openness of a family of torsion free sheaves. Jour. Math. Kyoto Univ. 16 (1976), 627-637.

[Nev] T. Nevins, Descent of coherent sheaves and complexes to geometric invariant theory quotients, Jour. Algebra 320 (2008), 2481-2495.

[New] P. E. Newstead, Introduction to Moduli problems and orbit spaces, TIFR lecture Notes, SpringerVerlag (1978).

[Ra] S. Ramanan, The moduli spaces of vector bundles over an algebraic curve, Math. Ann. 200 (1973), 69-84.

[Se] C. S. Seshadri, Fibrés vectoriels sur les courbes algébriques, Astérisque 96 (1982) 1-209.

School of Mathematics, Tata Institute of Fundamental Research, Homi Bhabha Road, Mumbai 400005, India

E-mail address: usha@math.tifr.res.in

School of Mathematics, Tata Institute of Fundamental Research, Homi Bhabha Road, MumBai 400005, INDIA

E-mail address: indranil@math.tifr.res.in 